\title{
Recombinant angiopoietin-1 restores higher-order architecture of growing blood vessels in mice in the absence of mural cells
}

\author{
Akiyoshi Uemura, ${ }^{1,2}$ Minetaro Ogawa, ${ }^{1}$ Masanori Hirashima, ${ }^{1}$ Takashi Fujiwara, ${ }^{3}$ \\ Shinji Koyama, ${ }^{2}$ Hitoshi Takagi, ${ }^{2}$ Yoshihito Honda, ${ }^{2}$ Stanley J. Wiegand, ${ }^{4}$ \\ George D. Yancopoulos, ${ }^{4}$ and Shin-Ichi Nishikawa ${ }^{1,5}$ \\ ${ }^{1}$ Department of Molecular Genetics, and \\ ${ }^{2}$ Department of Ophthalmology and Visual Sciences, Kyoto University Graduate School of Medicine, Kyoto, Japan \\ ${ }^{3}$ Laboratory Animal Center, Ehime University School of Medicine, Ehime, Japan \\ ${ }^{4}$ Regeneron Pharmaceuticals Inc., Tarrytown, New York, USA \\ ${ }^{5}$ Stem Cell Research Group, RIKEN Center for Developmental Biology, Kobe, Japan
}

Interactions between endothelial cells (ECs) and perivascular mural cells (MCs) via signaling molecules or physical contacts are implicated both in vascular remodeling and maintenance of vascular integrity. However, it remains unclear how MCs regulate the morphogenic activity of ECs to form an organized vascular architecture, comprising distinct artery, vein, and capillary, from a simple meshlike network. A clear elucidation of this question requires an experimental model system in which ECs are separated from MCs and yet form vascular structures. Here we report that injection of an antagonistic $\mathrm{mAb}$ against PDGFR- $\beta$ into murine neonates provides such an experimental system in the retina by completely blocking $\mathrm{MC}$ recruitment to developing vessels. While a vascular network was formed even in the absence of MCs, it was poorly remodeled and leaky. Using this vascular system ideal for direct assessment of the activities of MC-derived molecules, we show that addition of recombinant modified angiopoietin-1 restored a hierarchical vasculature, and also rescued retinal edema and hemorrhage in the complete absence of MCs. These observations demonstrate the potential of Ang1 as a new therapeutic modality for MC dropout in diseases such as diabetic retinopathies.

J. Clin. Invest. 110:1619-1628 (2002). doi:10.1172/JCI200215621.

\section{Introduction}

The first vascular plexus in the embryo is formed in the absence of perivascular mural cells (MCs; pericytes around capillaries and smooth muscle cells around larger vessels) and then rapidly remodels to a higherorder structure after the recruitment of MCs to the vascular network (1). For this vascular remodeling process, it has been widely accepted that interactions between endothelial cells (ECs) and MCs are critical cellular events (2), and a number of distinct signaling molecules have been implicated in EC-MC interactions (3, 4). Among the better characterized of these are

Received for publication April 5, 2002, and accepted in revised form September 24, 2002.

Address correspondence to: Akiyoshi Uemura, Department of Molecular Genetics, Kyoto University Graduate School of Medicine, 53 Kawahara-cho, Shogoin, Sakyo-ku, Kyoto, 606-8507 Japan. Phone: 81-75-751-4162; Fax: 81-75-751-4169;

E-mail: auemura@kuhp.kyoto-u.ac.jp.

Conflict of interest: S.J. Wiegand and G.D. Yancopoulos are employees of Regeneron Pharmaceuticals Inc., which retains all rights and financial interests in angiopoietin-1.

Nonstandard abbreviations used: mural cell (MC); endothelial cell (EC); angiopoietin-1 (Ang1); recombinant modified angiopoietin-1 (Ang1*); postnatal day $0(\mathrm{P} 0)$; platelet-endothelial cell adhesion molecule-1 (PECAM-1); $\alpha$-smooth muscle actin, (SMA); bromodeoxyuridine (BrdU). angiopoietin-1 (Ang1) and its receptor, Tie2, and PDGF-B and its receptor, PDGFR- $\beta(2,5-8)$. These molecules appear to be the best candidates for mediating EC-MC interactions, as these ligands (PDGF-B and Ang1) and their corresponding receptors (PDGFR- $\beta$ and Tie2) are expressed in a complementary manner, PDGF-B and Tie 2 in ECs, and PDGFR- $\beta$ and Ang1 in MCs (9-15). In addition, defects in either PDGF-B/PDGFR- $\beta$ or Ang1/Tie2 signaling result in vascular dysmorphogenesis and substantial reduction of MC association with blood vessels (14-22). It has been widely accepted that PDGF-B promotes MC recruitment to developing vessels by stimulating $\mathrm{MC}$ proliferation and migration (8). On the other hand, despite accumulating evidence for multiple roles of Ang1 in EC activities, including sprouting, survival, or antipermeability effects (6), the exact requirement of Ang1 for EC morphogenic activity in construction of hierarchical vascular structures is still unclear. Based upon the results of gene targeting studies, it was initially proposed that Ang1 indirectly facilitates MC recruitment to nascent endothelial tubes by stimulating the release of PDGF-B from ECs (23). The recruited MCs in turn direct the remodeling of the primitive vascular network to a higher-order struc- 
ture. Alternatively, recent studies have suggested that Ang $1 /$ Tie 2 signaling directly modulates numerous EC activities, thereby ensuring tight association with adjacent MCs $(24,25)$.

A substantial problem underlying previous studies, including genetic manipulations, is distinguishing the direct and indirect effects of Ang1 on ECs, as ECs and MCs mutually interact through multiple molecules in developing vessels, and such molecules may compensate for the altered Ang1 functions. A more definitive elucidation of the specific activity of Ang1 might be best evaluated in an experimental model system in which the vascular network is formed in the complete absence of $\mathrm{MC}$ association, thereby allowing the direct assessment of the particular activities of distinct MC-derived molecules.

While such a situation occurs in the early embryo, it is difficult to manipulate. The murine retinal vascular system, on the other hand, develops after birth (26), and has advantages in accessibility and manipulability. Here, we investigated the retinal vascular system as a suitable model for studying vascular development. In the developing retina, all vessels were covered by PDGFR- $\beta^{+}$MCs, be it artery, vein, or capillary. Systemic administration of an antagonistic mAb against PDGFR- $\beta$ during neonatal stages completely blocked $\mathrm{MC}$ recruitment to developing retinal vessels. Using this system, in which ECs are separated from MCs and yet form vascular structures, we assessed the potential of Ang1 to promote vascular remodeling in the absence of direct EC-MC interactions. Some severe defects in vascular remodeling and vessel integrity were largely corrected by the addition of recombinant Ang1 (Ang1*, modified from the native form), indicating that Ang1 is a key MC-derived regulator required for normal postnatal development and formation of the hierarchical microvasculature in the mouse retina. However, incomplete restitution of the vasculature indicates the involvement of other MC-derived molecules in the finetuning of vascular networks.

\section{Methods}

Animals. All animal procedures described in this study were performed using ICR mice (SLC Japan, Shizuoka, Japan) in accordance with the guidelines for animal experiments at Kyoto University Graduate School of Medicine.

$A b$ 's and factors. The mAbs against murine PDGFR- $\beta$ (clone APB5) (27) and murine Tie2 (clone TEK4) (28), and the fusion protein consisting of the extracellular domain of murine Tie 2 and the $\mathrm{F}_{\mathrm{c}}$ portion of human IgG (Tie2-Fc) (29), were prepared and labeled as previously described. Ang1* (Regeneron Pharmaceuticals Inc., Tarrytown, New York, USA) was prepared as previously described $(12,30)$. Ang $1 *$ is a recombinant Ang1 with a modified $\mathrm{NH}_{2}$-terminus in which the first 73 amino acids of human Ang2 are fused to the portion of human Ang1 beginning at the 77th amino acid residue, and amino acid replacement of $\mathrm{Cys}_{265}$ to Ser.
Labeled or unlabeled mAbs for platelet-endothelial cell adhesion molecule-1 (PECAM-1, clone MEC13.3 from Pharmingen, San Diego, California, USA; and clone $2 \mathrm{H} 8$ from Chemicon International, Temecula, California, USA), $\alpha$-smooth muscle actin (SMA) (clone 1A4; Sigma-Aldrich, St. Louis, Missouri, USA), fibronectin (clone FN-15; Sigma-Aldrich), desmin (clone D33; DAKO Corp., Glostrup, Denmark), and polyclonal Abs against laminin and type IV collagen (Cosmo Bio LSL, Tokyo, Japan) were purchased.

Injection of reagents. To block PDGFR- $\beta$ function, 100 $\mu \mathrm{g}$ of APB5 was injected intraperitoneally daily to neonatal mice beginning on postnatal day $0(\mathrm{P} 0)$. Littermates injected with nonspecific rat IgG (SigmaAldrich) or with vehicle alone served as controls. For intraocular injections, $1 \mu \mathrm{g}$ of Tie $2-\mathrm{Fc}$ or $300 \mathrm{ng}$ of Ang1* in $300 \mathrm{nl}$ sterile buffer was injected into the vitreous humor using glass capillary pipettes with a micromanipulator (Drummond Scientific Co., Broomall, Pennsylvania, USA) on days P1, P4, and P7. Control injections of BSA or human IgG Fc fragment (Jackson ImmunoResearch Laboratories, West Grove, Pennsylvania, USA) were given into contralateral eyes to ensure that vascular phenotypes were not due to injection artifacts.

Immunochemical and histological analysis. For wholemount preparations, the retinal cup was dissociated from other parts of the eye and used in immunochemical procedures as previously described (31). For cryosections, eyes were frozen in OCT compound (Sakura Finetek, Torrance, California, USA) and sectioned at a thickness of $10 \mu \mathrm{m}$. For hematoxylin and eosin (H\&E) staining, eyes were embedded in paraffin and sectioned at $5 \mu \mathrm{m}$ thickness. Confocal microscopy was performed using a Leica laser scanning microscope and TCS NT image software (Leica Microsystems Inc., Wetzlar, Germany). Transmission and scanning electron microscopy were performed under conditions optimized for analyzing vascular systems as previously described (32). The specimens were photographed with a Hitachi H-800 transmission microscope and S-800 scanning electron microscope (Hitachi Ltd., Tokyo, Japan).

Detection of cell proliferation and apoptosis. To label cells in $S$ phase of the cell cycle, an intraperitoneal injection (100 $\mu \mathrm{g} / \mathrm{g}$ body weight) of bromodeoxyuridine (BrdU) was given 1 hour before enucleation. The incorporated BrdU was detected by immunohistochemistry using an FITC-conjugated mAb for BrdU (Roche Molecular Biochemicals, Mannheim, Germany) according to the manufacturer's instructions. TUNEL technique was used to detect apoptotic cells according to the standard protocol described by Gavrieli et al. (33). Dying cells were also labeled in vivo using propidium iodide (Molecular Probes Inc., Eugene, Oregon, USA), a fluorescent molecule that is excluded from uninjured viable cells and binds to the DNA and RNA of transiently injured viable cells or cells undergoing either necrosis or apoptosis. One hour after an intraperitoneal injection of 
propidium iodide ( $25 \mu \mathrm{g} / \mathrm{g}$ body weight), retinas were dissected and examined by fluorescence microscopy.

Visualization of vascular leakage. Ten minutes after an injection of 4.4-kDa TRITC-dextran solution $(50 \mu \mathrm{l}$ at $10 \mathrm{mg} / \mathrm{ml}$ in PBS; Sigma-Aldrich) into the heart over the chest wall, eyes were enucleated and extravasated TRITC-dextran in the retina was observed by fluorescence microscopy.

Quantitative analysis. Flat-mount retinas stained with PECAM-1 were photographed on an Axiophot microscope (Carl Zeiss Jena GmbH, Jena, Germany) equipped with a charge-coupled device camera and associated software (AxioVision version 3.1). The images were processed using NIH Image software (version 1.62). Vascular density was quantified by measuring PECAM-positive vessels in a fixed area photographed using a $10 \times$ objective. The radius of vascular networks was quantified by measuring the length between the center of the optic nerve and the edge of leading vessels. These measurements were performed in four quadrants and then averaged for each retina. For each treatment group, six retinas were analyzed. The data were expressed as mean \pm SD and were statistically analyzed by Student $t$ test. Differences were considered statistically significant when $P$ values were below 0.05 .

\section{Results}

$M C$ recruitment in postnatal development of the retinal vasculature. Previously, using SMA as a marker for MCs, MC coverage of the developing retinal vessels was reported to proceed by MC outmigration along arteries and to lag many days behind capillary formation (34). Based on this observation, it was hypothesized that remodeling occurs only in the MC-free endothelial plexus, and such plasticity is lost upon the acquisition of an MC coating. This hypothesis is challenged, however, by findings that MCs around capillaries are negative for $\operatorname{SMA}(15,35)$. To reassess the relationship between retinal vascular remodeling and MC recruitment, we combined PDGFR- $\beta$, desmin, and SMA as MC markers. PECAM-1 was used as an EC marker. At postnatal day 1 (P1), a primitive vascular plexus begins to sprout radially from the optic disc located in the center of the retina. As vessels spread toward the periphery, the primitive vascular network is gradually transformed into higher-order architecture comprised of distinctive arteries, veins, and capillaries. In the developing retina, PDGFR- $\beta$ was expressed only around the spreading vessels, and not in other cellular components (Figure 1, a and b). Throughout the course of retinal vascular development, PDGFR- $\beta$ expression was detected around all developing vessels

\footnotetext{
Figure 1

MC recruitment in postnatal development of the retinal vasculature. ( $\mathbf{a}$ and $\mathbf{b}$ ) Counterstaining of P5 retina with hematoxylin and PECAM (a) or PDGFR- $\beta(\mathbf{b})$. In the retina, PDGFR- $\beta$ is expressed only around developing vessels (arrowheads) arising from the optic nerve $(\mathrm{ON})$. (c-n) Double immunofluorescent staining using PECAM, PDGFR- $\beta$, desmin, and SMA. From the beginning of vascular development, PDGFR- $\beta$ is expressed around the entire retinal vascular network (c), except for sprouting tips (arrowheads in d). PDGFR- $\beta$ is expressed in MCs located in the outer linings of endothelial surfaces (e). Desmin is expressed in all types of retinal vessels ( $\mathbf{f}$ ), including the growing vascular edges $(\mathbf{g})$, with filamentous patterns in PDGFR- $\beta^{+}$MCs (arrowheads in $\mathbf{h}$ ). SMA expression over the primary vascular plexus (i and $\mathbf{j}$ ) is maintained in arterial walls and fades in venous and capillary walls as vascular remodeling proceeds (k). Finally, SMA expression in MCs is confined to arteries ( $\mathbf{I}$ and $\mathbf{m}$ ) and newly formed vessels in the advancing regions (n). (o-q) Scanning electron microscopy demonstrating morphological heterogeneity of MCs in accordance with the types of vessels: multiconcentric MCs around arteries (o), singlelayer MCs around veins ( $\mathbf{p}$ ), and capillary pericytes with long processes oriented longitudinally along the axis of vessel walls (arrowhead in $\mathbf{q}$ ).
}
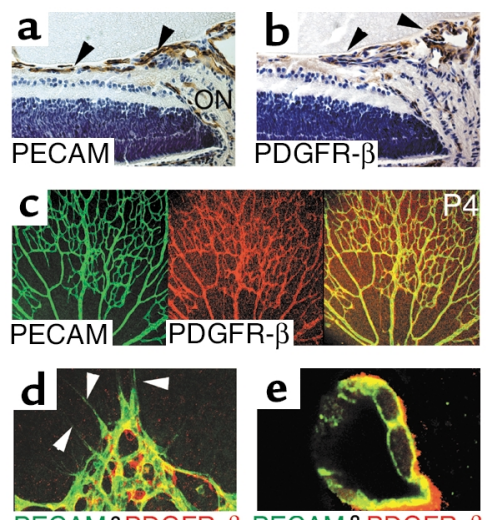

PECAM\&PDGFR- $\beta$ PECAM\&PDGFR- $\beta$
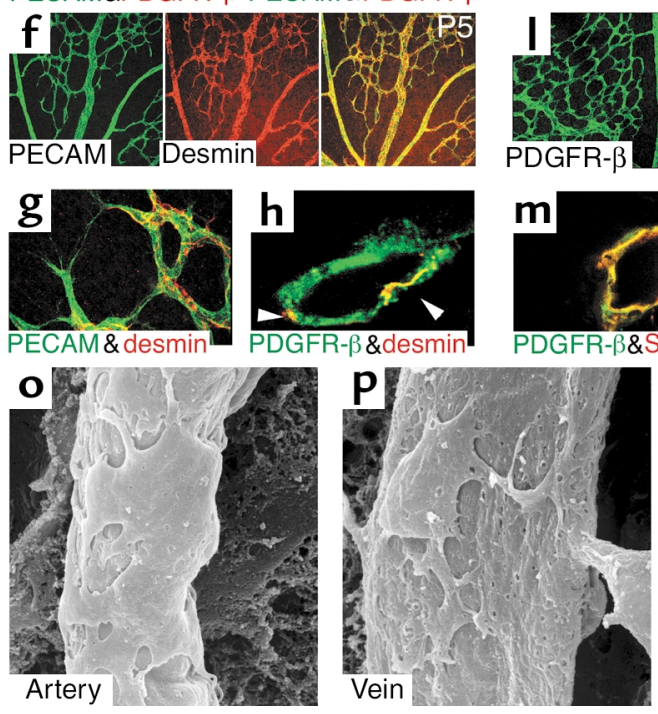
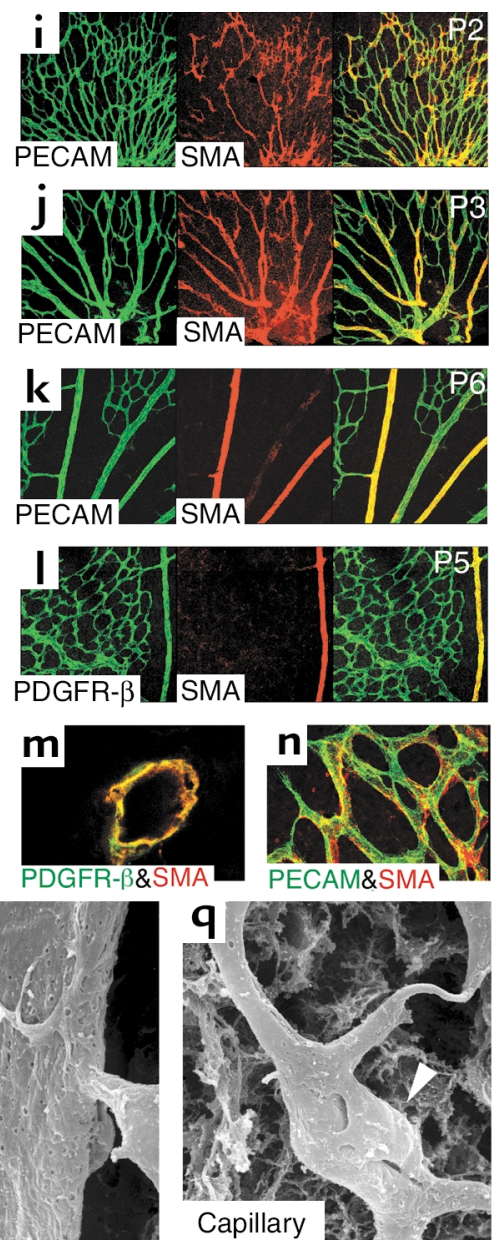

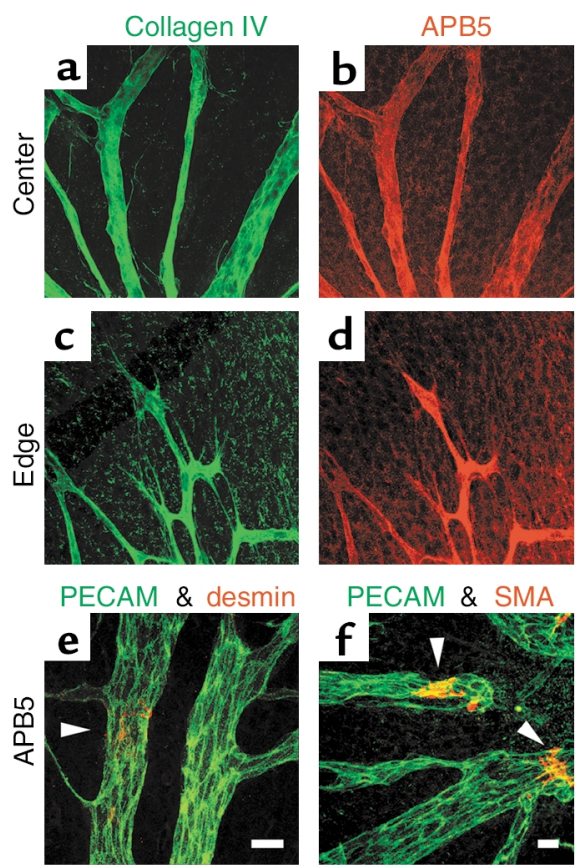

PECAM \& SMA
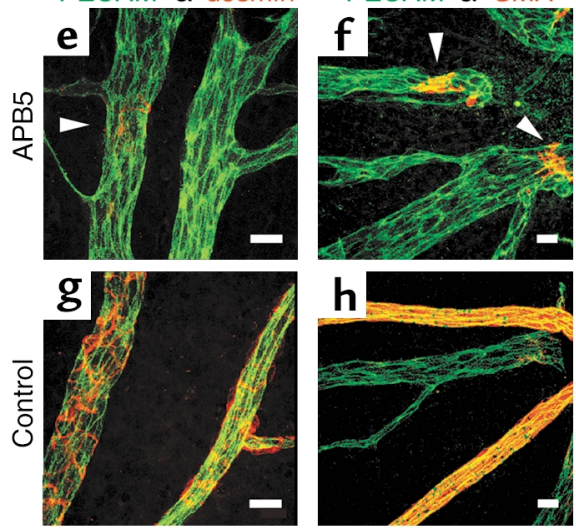

h
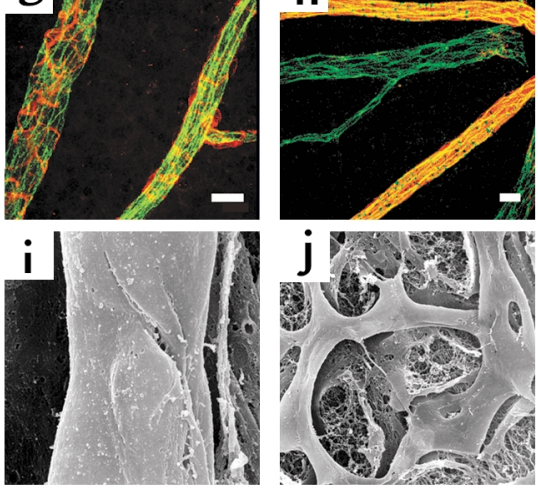

(Figure 1c) except the sprouting tips (Figure 1d). Highmagnification views of transverse sections revealed that PDGFR- $\beta$ was expressed in MCs intimately associated with EC tubes (Figure 1e). Desmin was expressed around all retinal vessels (Figure 1f), even at growing vascular edges (Figure 1, g and h). Surprisingly, in contrast to a previous report (34), we observed that SMA was expressed by MCs associated with most of the developing retinal vessels at early stages (P2 and P3) of development (Figure 1, i and j). Later, as vascular remodeling proceeded, SMA expression was progressively lost in MCs associated with differentiating capillaries and veins (Figure 1k). Eventually, strong SMA expression was detected only in MCs around arteries (Figure 1, 1 and $\mathrm{m}$ ). In areas of peripheral advancing vessels, however, SMA was still expressed around newly formed vessels even at later stages (Figure 1n). Scanning electron microscopy also demonstrated that MCs were associated with all types of retinal vessels, be it artery (Figure 1o), vein (Figure 1p), or capillary (Figure 1q), despite apparent morphological heterogeneity. Thus MCs are recruited to all types of developing ves-

\section{Figure 2}

Inhibition of MC recruitment to retinal vessels by postnatal treatment with anti-PDGFR- $\beta$ Ab. (a-d) Distribution of systemically administered APB5 in the retina. APB5 (rat anti-mouse PDGFR- $\beta$ mAb) was labeled with anti-rat $A b 3$ hours after an intraperitoneal injection into a P4 neonate. Distribution of APB5 ( $\mathbf{b}$ and $\mathbf{d}$ ) coincided with the retinal vascular networks, as illustrated by type IV collagen ( $\mathbf{a}$ and $\mathbf{c}$ ). (e-h) Double immunofluorescent staining of PECAM and desmin at P5 (e and $\mathbf{g}$ ), and PECAM and SMA at P8 ( $\mathbf{f}$ and $\mathbf{h}$ ). In contrast to the successive MC coverage in the control ( $\mathbf{g}$ and $\mathbf{h}$ ), APB5-treated mice failed to recruit MCs in the developing retina, with the exception of the continued presence of a few MCs (arrowheads) only at the roots of trunk vessels arising from the optic disc (e and $\mathbf{f}$ ). Bars represent $20 \mu \mathrm{m}$. (i and $\mathbf{j}$ ) Scanning electron microscopy depicting denuded endothelial tubes with complete absence of MC coverage in large vessels (i) and capillaries (j) in APB5-treated retina.

sels in the retina, but exhibit distinct immunochemical and histological features that are characteristic of the types of vessels with which they are associated.

Inbibition of $M C$ recruitment to retinal vessels by postnatal treatment with anti-PDGFR- $\beta$ Ab. Although previous gene targeting studies indicated critical roles for PDGF-B/PDGFR- $\beta$ in MC recruitment, the dependence of MC recruitment on PDGF-B/PDGFR- $\beta$ appears variable among tissues $(14,15)$. As virtually all MCs in retinal vessels express PDGFR- $\beta$, we next investigated the possibility that MC recruitment to retinal vessels might be blocked completely by administration of an antagonistic anti-PDGFR- $\beta$ mAb (APB5) (27). After preliminary experiments, we used a regimen in which neonates, beginning on $\mathrm{P} 0$, were treated with daily intraperitoneal injections of $100 \mu \mathrm{g}$ APB5. Because of the inherent property of this $\mathrm{mAb}$, continuous injection of such a high dose is required to block PDGFR- $\beta$ completely (27). Accessibility of APB5 injected intraperitoneally into the retina of neonates was confirmed by its distribution over the entire retinal vascular network (Figure 2, a-d). Neonates treated with APB5 grew up healthy and had normal weight gain and appearance. In retinas of APB5-treated mice, all developing vessels were completely devoid of MCs, with the exception of the continued presence of a few MCs in the stems of vessels at the optic disc (Figure 2, e and f). Endothelial tubes, both in large vessels (Figure 2i) and capillaries (Figure $2 \mathrm{j}$ ), were entirely denuded of MC coatings. In contrast, MCs were successfully recruited to the nascent vessels in control mice (Figure 2, $\mathrm{g}$ and h) and in mice injected with anti-PDGFR- $\alpha$ antagonistic mAb (APA5) (36) (data not shown). Thus, the PDGFR- $\beta$ signal is essential for MC recruitment to developing retinal vessels.

Phenotype of retinal vessels that lack MCs. In control retinas, five to six arterial trunks were usually formed, which were characterized by progressive loss of direct anastomoses with the capillary plexus (Figure 3 , a and c). Between the arterial trunks were venous trunks that maintained extensive anastomoses with the capillary plexus. The venous trunks were always larger in size 
than the arterial trunks. In the APB5-treated mice, on the other hand, while the superficial plexus of retinal vessels initially continued to grow toward the periphery of the retina and underwent remodeling into large and small vessels, the architecture of this MC-free vascular system was severely distorted (Figure 3, b and d). All types of retinal vessels were enlarged and anastomosed randomly with each other. Arterial and venous trunks frequently crossed each other and were connected directly via short vessels, which were never seen in control retinas. These MC-free vessels were perfused with circulating blood, since they were labeled in vivo by systemically perfused lectin (data not shown). Interestingly, although the normal morphological distinctions between artery and vein were largely absent, arteries were still smaller than veins, and some degree of capillary pruning remained around the major arteries (Figure 3d). In addition, the intensity of PECAM staining remained stronger in arteries than in veins, as is normally the case. Thus, some properties distinguishing arteries and veins can be established in the absence of normal interactions with MCs.

At around $\mathrm{P} 8$ to $\mathrm{P} 9$, advancing vessels were present at the edges of the retina in control mice (Figure 3a), while in the APB5-treated mice, the peripheral retina remained unvascularized (Figure $3 \mathrm{~b}$ ). This impression was quantitatively assessed by measuring the radius of

\section{Figure 3}

Disorganized vascular remodeling in the absence of MCs. ( $\mathbf{a}$ and $\mathbf{b}$ ) Double immunostaining with PECAM (brown) and SMA (blue) of P8 retina. In the control (a), advancing vessels had reached the edges of the retina, while in the APB5-treated mice, the peripheral retina remained unvascularized $(\mathbf{b})$. Note the strong SMA expression around arteries in the control retina. (c and d) PECAM staining of $\mathrm{P} 8$ retina of control (c) and APB5-treated mice (d). Note the remaining capillary pruning and intense PECAM staining in arteries even in the absence of MCs. (e and $\mathbf{f}$ ) Morphometric analysis of retinal vasculature at P3, P5, and P8. Black bars, control mice; gray bars, APB5treated mice. Data (mean $\pm S D$ ) are expressed as percentages relative to the control retina on $\mathrm{P} 3 .{ }^{*} P<0.05$ by Student $t$ test. ( $\mathbf{g}$ and $\mathbf{h}$ ) High-magnification view of PECAM staining in P5 retina. The shape of each EC is illustrated by white lines. Increases in the number and size of ECs contributed to vessel enlargement in the absence of MCs. Bars represent $20 \mu \mathrm{m}$. (i and $\mathbf{j}$ ) Detection of dying ECs by TUNEL assay (i) and in vivo propidium iodide labeling ( $\mathbf{j}$ ) in P5 retina. Endothelial apoptosis (arrowheads) was not accelerated even in the absence of MCs. (k-n) Double labeling of BrdU-incorporating cells (green) and PECAM+ ECs (red) in P5 retina. ECs were mitotically active, particularly in the regions of advancing retinal vessels in both APB5-treated mice ( $\mathbf{m}$ and $\mathbf{n}$ ) and controls ( $\mathbf{k}$ and $\mathbf{I})$. A, artery; $\mathrm{V}$, vein. the retinal vasculature. In addition, to evaluate the morphometric changes, we quantified the vascular density in the proximal vascularized retina. At $\mathrm{P} 3$, there were no significant differences in either of these parameters between control and APB5-treated mice. However, at later stages of retinal development, reduced radius and increased density became apparent in the retinal vasculature of APB5-treated mice. The radius was reduced by $23 \% \pm 4.1 \%$ at $P 5$, and by $38 \% \pm 5.1 \%$ at P8 (Figure 1e; $n=6, P<0.05$ for each), while the density was increased by $31 \% \pm 11 \%$ at P5 and by $93 \% \pm 20 \%$ at P8 (Figure 1f; $n=6, P<0.05$ for each). The substantial increase of retinal vascular density despite the apparently sparse capillary meshwork was ascribed to enlargement of both trunk and capillary vessels. In these enlarged vessels, ECs were increased in number as well as in size (Figure 3 , g and $\mathrm{h}$ ).

Next, we assessed the rate of apoptosis or proliferation of retinal ECs, as MCs were proposed to inhibit endothelial proliferation $(37,38)$ or promote endothelial survival $(34,39)$. Even in the absence of MCs, no significant increase of apoptotic ECs was observed by TUNEL (Figure 3i) or in vivo propidium iodide labeling assays (Figure 3j). Moreover, we could not detect significant changes in the number of BrdU-labeled ECs (Figure 3, k-n), which were present particularly in the advancing vessels at the periphery of the retina, indi-

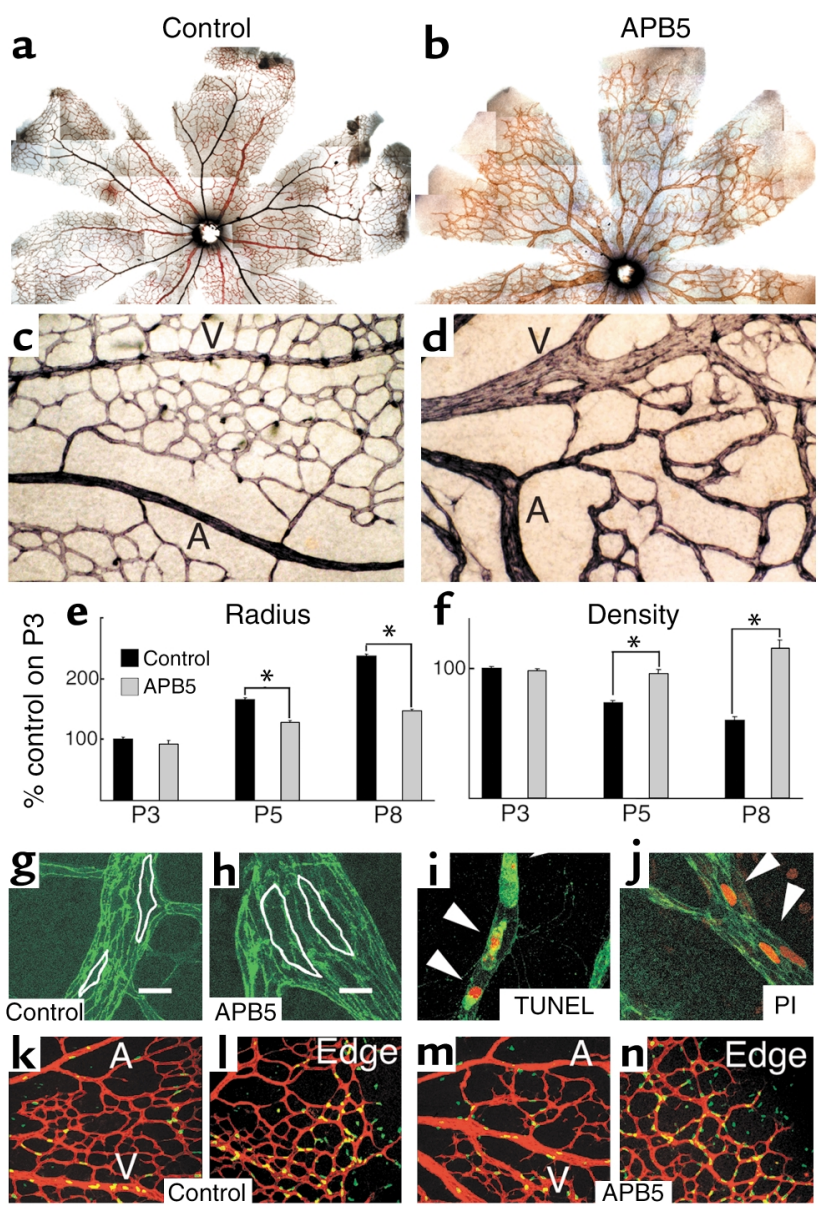



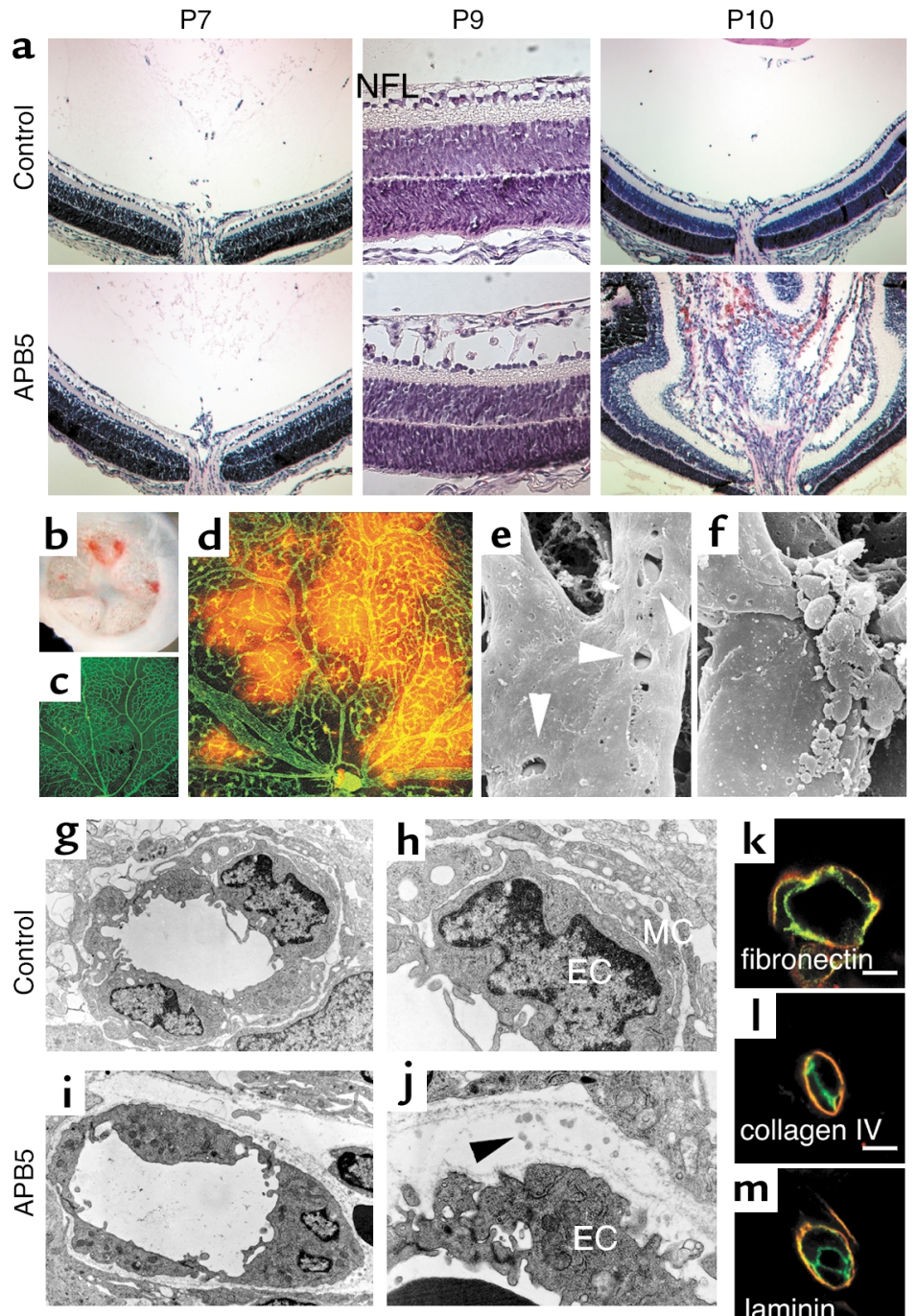

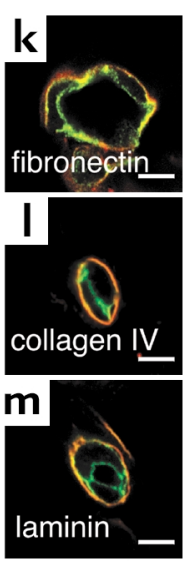

\section{Figure 4}

Deteriorated integrity of retinal vessels in the absence of MCs. (a) H\&E staining of retinal sections of control (top) and APB5-treated (bottom) mice. APB5 treatment induced progressive exacerbation of retinal edema in the nerve fiber layer (NFL) at around P8 to $P 9$, resulting in retinal collapse and massive hemorrhage at P10. (b) Retinal cup of APB5-treated mouse at P9 depicting widespread hemorrhage and funnel-shaped retinal collapse. (c and $\mathbf{d}$ ) Visualization of vascular leakage in P8 retina by TRITC-dextran perfusion. Multifocal leakage of systemically perfused dextran demonstrated the increased vascular permeability in the absence of MCs (d). No leakage was found in the control retina (c). (e and $\mathbf{f}$ ) Scanning electron microscopy demonstrating numerous cleft formations (arrowheads in $\mathbf{e}$ ) in denuded endothelial sheets and extravasation of blood cells $(\mathbf{f})$. $(\mathbf{g}-\mathbf{j})$ Ultrastructural analysis depicting vessel walls with $M C$ coverage in the control retina ( $\mathbf{g}$ and $\mathbf{h}$ ) and $\mathrm{MC}$-free endothelial walls in the APB5-treated retina (i and $\mathbf{j}$ ). In the absence of MCs, extraluminal spaces were enlarged with fluid accumulation. Note the swelling of periendothelial basement membranes with cell debris (arrowhead in $\mathbf{j}$ ). EC, endothelial cell; MC, mural cell. (k-m) Double immunofluorescent staining of PECAM (green) and fibronectin (k), type IV collagen (I), and laminin ( $\mathbf{m})$ (red) in retinal vessels of APB5-treated mice. Major extracellular matrix components of basement membranes were deposited around EC surfaces in the absence of MCs. Bars represent $5 \mu \mathrm{m}$. cating that new ECs are constitutively generated. Thus, the morphogenic defect in the retinal vessels of APB5treated mice does not appear to be attributable to changes in proliferation or apoptosis of ECs.

In addition to the failure in retinal vascular remodeling, APB5 treatment induced progressive edema at around $\mathrm{P} 8$ to $\mathrm{P} 9$ in the nerve fiber layer across which the retinal vasculature extends, finally resulting in funnelshaped retinal collapse and massive intraocular hemorrhage at P10 (Figure 4, a and b). As PDGFR- $\beta$ is expressed only in MCs in the developing retina, APB5induced changes in the retinal architecture appeared to be due to the impaired vascular function resulting from the absence of MCs. Indeed, multifocal leakage of systemically perfused dextran confirmed that the edema and hemorrhage was caused by an elevated leakiness of the retinal vascular system (Figure 4, $c$ and d). Multiple clefts that formed in denuded endothelial sheets (Figure 4e) suggested a histological basis for extravasation of plasma and blood cells (Figure 4, $\mathrm{f}$ and i). In addition, the area occupied by periendothelial basement membranes was expanded (Figure 4, $i$ and $j$ ), while in the con- trol retinas, basement membranes formed a fine layer around vessel surfaces (Figure 4, $g$ and $h$ ). These findings are similar to the histopathological features of diabetic retinopathy, where selective loss of pericytes in microvessels is an early pathological change associated with retinal microaneurysms, edema, and hemorrhage $(40,41)$. Deposition of fibronectin (Figure $4 \mathrm{k}$ ), type IV collagen (Figure 4l), and laminin (Figure $4 \mathrm{~m}$ ) around EC surfaces of MC-free vessels indicated that the ECs by themselves can produce major extracellular matrix components of basement membranes.

Substantial replacement of $M$ C activity by Ang1* Given that the defects of the retinal vascular system in ABP5-treated mice are due to the failure of MCs to associate with developing vessels, this experimental model provides an ideal system to assess the direct effects of MC-derived molecules on the control of EC morphogenic activity.

First, to examine the physiological role of Ang1/Tie2 signaling in developing retinal vessels, where all ECs express Tie 2 receptor (Figure 5a), we injected Tie2-Fc (Figure 5c) or recombinant Ang1* (Figure 5d) into the vitreous cavity at $\mathrm{P} 1$ and analyzed the retinal vessels at $\mathrm{P} 4$. 
In eyes treated with Tie2-Fc, the retinal vascular system was comprised of homogeneously sized capillaries and lost the hierarchical architecture. This defect in formation of large vessels is consistent with previous reports on mice null for Tie2 (17) or Ang1 (18). In clear contrast, Ang $1 *$ injections accelerated (though only slightly) the rate of vascular development by increasing the radius of vascular networks $(16 \% \pm 12 \%, n=6, P=$ not significant) (Figure $5 \mathrm{i}$ ) and decreasing vascular density $(10 \% \pm 13 \%$, $n=6, P=$ not significant) (Figure $5 \mathrm{j}$ ). The most striking effects of Ang1* were an increase in the number of arterial and venous trunk vessels (control vs. Ang1*, respectively: artery, $5.8 \pm 0.8$ vs. $7.9 \pm 0.9$; vein, $6.0 \pm 0.9$ vs. $9.0 \pm 0.8$; $n=6, P<0.05$ for each) and increased vascular diameter (percent increase in control vs. Ang $1^{*}$, respectively: artery, $32 \% \pm 19 \%$; vein, $36 \% \pm 21 \% ; n=12, P<0.05$ for each) (Figure $5, \mathrm{k}$ and $\mathrm{l})$. These results indicate critical involvement of Ang1 in retinal vascular remodeling, especially in generation and enlargement of trunk vessels.

Next we assessed the effects of Ang1* on vascular remodeling in the absence of MCs. Even after depletion of MCs, all ECs still expressed Tie2 receptor (Figure 5e). Strikingly, APB5-induced defects in retinal vasculature (Figure $5 \mathrm{f}$ ) were substantially restored by Ang1* injection (Figure $5 \mathrm{~g}$ ), though MC recruitment remained uncorrected (Figure $5 \mathrm{~h}$ ). This restoration represents Ang1 acting on ECs to regulate their morphogenic processes. However, compared with the normal retina, there remained clear differences in the vascular structure of retina treated with both Ang1* and APB5. Although the radius of the vascular networks was the same as in the normal retina (Figure 5i), all types of vessels were significantly enlarged (percent enlargement in control vs. APB5 + Ang $1^{*}$, respectively): (artery, $65 \% \pm 49 \%, n=12, P<0.05$; vein, $57 \% \pm 50 \%$, $n=12, P<0.05$; capillary, $57 \% \pm 38 \%, n=24, P<0.05)$ (Figure 5, k-m). Vessel enlargement together with formation of numerous capillaries resulted in significantly higher vascular density (increase of $57 \% \pm 12 \%$, $n=6, P<0.05)$ (Figure 5j).

At later stages (P8) after repeated Ang1* injections, restoration of higher-order architecture to the retinal vasculature became more evident (Figure 6c). Arteries were more clearly distinguishable from veins by their size as well as the intensity of PECAM staining. However, some aspects of the vascular structure, such as vessel enlargement and excessive capillary formation, remained different from the normal retina (Figure 6a). Remarkably, anastomoses between capillaries and arteries were largely retained in retinas treated with both Ang1* and APB5 (Figure 6c). In addition to the effects on these morphological processes in vascular remodeling, Ang1* prevented retinal collapse by suppression of edema and hemorrhage, even at P10 (Figure 66), implicating a direct role for Ang1 in the maintenance of endothelial integrity. Indeed, extravascular fluid accumulation and swelling of basement membranes were corrected to normal levels (Figure 6, g and h).

All the above effects of Ang $1 *$ injections were abrogated by coinjection of Tie2-Fc (data not shown), indicating that they are Ang1-specific. These results reveal that Ang1 alone can ameliorate most of the defects in vascular patterning and vessel integrity caused by MC loss.

\section{Discussion}

In this study, we described a novel experimental system in which the vascular network is formed in the absence of MCs. This system allowed assessment of the direct effects of MC-derived molecules on various activities of ECs, including processes taking place

\footnotetext{
Figure 5

Effects of Ang1 on retinal vascular development. (a-g) $\mathrm{P} 4$ retinal vasculature stained with Tie2 ( $\mathbf{a}$ and $\mathbf{e})$ or with PECAM-1 (b-d, f, and $\mathbf{g}$ ). Normally, Tie2 is expressed in all retinal vessels (a). Intraocular injections of Tie2-Fc (c) and Ang1* (d) displayed clear contrasts in effects on vascular remodeling, especially in generation of trunk vessels. In the APB5-treated mice, Tie2 is still expressed in all retinal vessels even in the absence of MCs (e). Disorganized vascular architecture induced by systemic APB5 treatment (f) was ameliorated to a great extent by intraocular injections of Ang $1^{*}(\mathbf{g})$. (h) Scanning electron microscopy demonstrating complete absence of MCs in the retina treated with APB5 + Ang1*. (i-m) Quantitative analysis of the radius (i), density $(\mathbf{j})$, arterial diameter $(\mathbf{k})$, venous diameter $(\mathbf{I})$, and capillary diameter $(\mathbf{m})$ of retinal vasculature at P4. Data (mean $\pm \mathrm{SD})$ are expressed as percentages relative to the normal retina. ${ }^{*} P<0.05$ versus normal (Student $t$ test).
}
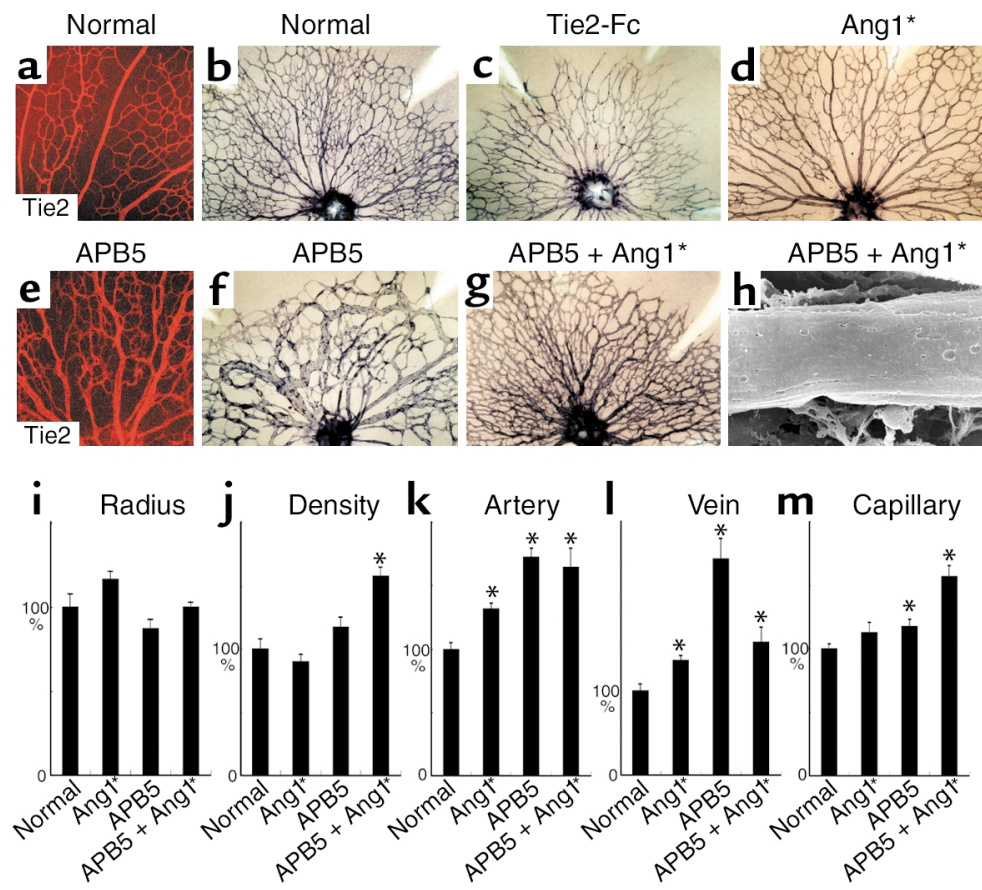

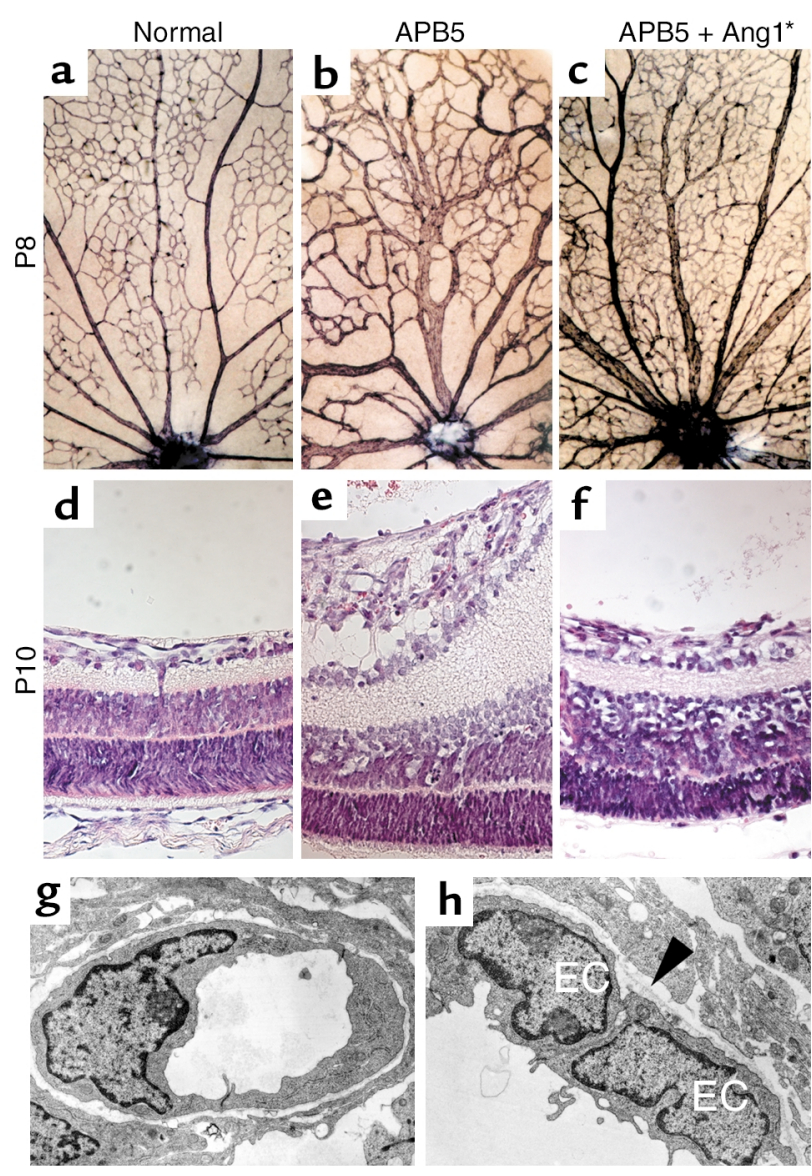

\section{Figure 6}

Restoration of hierarchical vascular architecture and vessel integrity by Ang $1^{*}$. (a-c) PECAM staining of the P8 retina of normal (a), APB5-treated (b), and APB5 + Ang1 ${ }^{*}$-treated (c) mice. Disorganized vascular remodeling induced by APB5 (b) was substantially restored by Ang ${ }^{*}$, with clearer distinctions between arteries and veins $(\mathbf{c})$. Note the formation of numerous capillaries and extensive anastomoses with arteries. (d-f) H\&E staining of P10 retina of normal (d), APB5-treated (e), and APB5 + Ang1*-treated (f) mice. Ang1* prevented APB5-induced retinal collapse by suppression of edema and hemorrhage. ( $\mathbf{g}$ and $\mathbf{h}$ ) Ultrastructural analysis of the vessel walls in the retina treated with APB5 + Ang1*. Despite the absence of MCs, extraluminal fluid accumulation almost completely disappeared $(\mathbf{g})$ and basement membranes were corrected to form a fine layer (arrowhead in $\mathbf{h}$ ) as seen in the normal vessels. Note the intact integrity of the EC-EC junctions. EC, endothelial cell.

during remodeling. To inhibit MC recruitment in the retina, we systemically injected an antagonistic $\mathrm{mAb}$ to PDGFR- $\beta$ (APB5), as PDGFR- $\beta$ signaling was implicated in MC recruitment to nascent vessels (14, $15,42)$. While systemic APB5 administration completely blocked MC recruitment in the retina, it did not cause dissociation of MCs from ECs in other regions, such as the brain (data not shown), where $M C$ recruitment was inhibited in embryos null for PDGF-B or PDGFR- $\beta(14,15)$. Moreover, APB5 administration to older mice has no effect on MCs in the retinal vessels (data not shown). Taken together, PDGFR- $\beta$ signaling is essential for recruiting MCs in developing vessels, but not for maintenance of EC$\mathrm{MC}$ association or MC survival in quiescent vessels.

Although the overall condition of the neonates appeared unimpaired by systemic APB5 treatment, postnatal vascular development in some tissues was affected to some extent. For instance, in the kidney, APB5 treatment inhibits new formation of glomeruli in the outer cortical layer, whereas mature glomeruli in medullar regions remains unaffected (43). Thus, the possibility that developmental failure in other tissues, such as the kidney, secondarily affects the processes in retinal vascular development should be considered. However, as direct intraocular injections of APB5 (which may not have systemic influence) resulted in retinal vascular changes similar to those induced by systemic injection (data not shown), the secondary effects of systemic injection on the retinal vasculature may be minimal. It should be noted that compared with the intraocular injection, administration of APB5 by systemic injection is technically easier and gives more reproducible results in complete inhibition of MC recruitment.

Consistent with functional analysis using APB5, immunostaining of retinal vessels with this $\mathrm{mAb}$ demonstrated that PDGFR- $\beta^{+}$MCs were associated with virtually all vessels from the early stages of retinal vascular development. This observation, however, contrasts with a previous model indicating that a considerable portion of developing retinal vessels, particularly veins and capillaries, remains unassociated with MCs for more than 10 days after birth (34). We think this discrepancy is due to the fact that the previous conclusion was drawn from experiments using only SMA as an MC marker. Recent studies using transgenic mice expressing $\beta$-galactosidase in MCs (44) or in situ hybridization with a probe against PDGFR- $\beta$ (45) are consistent with our present results showing that MCs are distributed along the entire retinal vasculature.

Taken collectively, we propose the following model for $\mathrm{MC}$ recruitment during retinal vascular development. First, nascent retinal vessels rapidly recruit PDGFR- $\beta^{+}$ MCs, which are regulated by EC-derived PDGF-B. While most newly recruited MCs are initially similar, they rapidly diversify, either through interactions with distinctive EC subsets or through the influence of regional hemodynamic constraints, thereby generating morphologically distinct MC populations at the appropriate sites in the vascular system. Likewise, SMA expression is determined extrinsically in response to specific conditions associated with arteries or nascent capillaries.

While vascular growth could proceed to a fair extent in the absence of MCs, the resulting vascular system was severely disorganized and leaky. This is consistent with the prevalent notion that MCs are required for both vascular remodeling and maintenance of the integrity of the EC layers $(2,46,47)$. It was previously suggested that in the processes of vascular remodeling, MCs regulate EC behavior through inhibiting EC mitotic activity as well as EC apoptosis, thereby pro- 
moting vessel stabilization. While APB5 treatment retarded radial extension of retinal vasculature and enhanced dilation of each vessel, we did not observe changes in apoptosis or proliferation of ECs in APB5treated retina. Thus, MCs may contribute to the remodeling process through regulating yet-unidentified activities of ECs, other than proliferation or survival.

The vascular network devoid of MCs, though defective, is useful for assessing the net activity of Ang1, which is constitutively expressed in developing retina (48). As remodeling is a morphogenic process, it is reasonable to think complex positional cues are required for this process. Despite our initial expectation that intraocularly injected Ang1*, which might be present diffusely over the retina, has only a limited ability to compensate for the loss of MCs, the organized vascular architecture was substantially restored by Ang $1^{*}$. In this experiment, we injected Ang $1 *$ in one eye while MC recruitment was inhibited in both eyes by systemic APB5 injection, thereby allowing the other eye to serve as control. The restoration of the retinal vascular architecture only at the Ang $1{ }^{*}$-injected side indicated that Ang $1^{*}$ acted directly on the retinal vessels rather than changing the organization of the systemic vascular system. It should be emphasized that Ang1* could not restore $\mathrm{MC}$ recruitment at all. Thus, this restitution is due to the direct action of Ang1* on ECs. Moreover, suppression of leakage of $\mathrm{MC}$-free vasculature indicates that Ang1 exerts its antipermeability effects $(49,50)$ directly through maintenance of EC integrity. Hence, the previous hypothesis that the principal role of Ang1 is to stabilize the physical association of MCs with ECs, thereby strengthening further EC-MC interactions (23, 51 ), should be reevaluated.

While our present study using recombinant Ang1* suggested a role for Ang1 as the major molecule responsible for MC-mediated vascular remodeling, further elucidation is required to understand the exact roles of Ang1 in physiological vascular development. Although Ang1* is thought to have activity similar to that of the native Ang1 in vitro, especially in activation of Tie2 receptor, it remains to be determined whether the modifications in Ang1* confer additional activities to or impair the original functions of native Ang1 during blood vessel formation $(52,53)$. Moreover, a recent study showing the direct binding of Ang1 to $\beta 1$-integrin suggests the possibility that the phenomena described here could be partly ascribed to this Tie2-independent pathway (54). Our present observations, however, give some possible insights into the processes through which Ang1 eventually guides the hierarchical patterning of developing vessels. First, increases in the number and size of trunk vessels by Ang1* treatment suggest the importance of Ang1 in generating major blood vessels. This role is consistent with increased frequency of enlarged vessel diameters in Ang1-overexpressing transgenic mice $(49,55)$ or with decreases in the number and size of large vessels in mice null for Ang1 (18). On the other hand, excessive vessel formation with retained periarterial capillaries in the absence of MCs indicates that Ang1 alone is not sufficient for normal vascular patterning, and that fine-tuning of the vascular network requires further involvement of MCs. As previously suggested (2), stabilization of EC integrity by Ang1 may render the vascular system permissive to other MCderived signals, and our experimental system will be useful in the identification of such molecules.

Finally, the present experimental system in which MCs are dissociated from the vascular network is useful for understanding various pathological conditions. In diabetic retinopathy, selective loss of MCs from microvessels has been suggested to initiate subsequent events such as aneurysm formation, retinal edema, and hemorrhage (40). However, the long course of diabetic retinopathy has hindered analysis of causal connections between these events. Similarities in the histological features of the vascular system between APB5treated retina and diabetic retinopathy indicate the potential of this experimental system - for instance, our results showing that Ang1* administration inhibited vascular leakage in the absence of MCs, predicting a possibility for use of Ang1 therapy to treat diabetic retinopathy. Indeed, a recent study has confirmed that Ang1 can ameliorate the pathology seen in rodent models of diabetic retinopathy (56).

\section{Acknowledgments}

We thank all the members of our laboratory for helpful discussions. We thank N. Takakura for generation of APA5 and APB5 hybridomas, and R. Yu and N.M. Rao for critical comments on the manuscript. This work is supported by grants from the Ministry of Education, Science, Sports and Culture of Japan (07CE2005, 12215071, and 12670301), the Cell Science Research Foundation, and the Japanese Society for the Promotion of Science, Research for the Future Program.

1. Risau, W. 1997. Mechanisms of angiogenesis. Nature. 386:671-674.

2. Yancopoulos, G.D., et al. 2000. Vascular-specific growth factors and blood vessel formation. Nature. 407:242-248.

3. Beck, L., Jr., and D'Amore, P.A. 1997. Vascular development: cellular and molecular regulation. FASEB J. 11:365-373.

4. Carmeliet, P. 2000. Mechanisms of angiogenesis and arteriogenesis. Nat. Med. 6:389-395.

5. Gale, N.W., and Yancopoulos, G.D. 1999. Growth factors acting via endothelial cell-specific receptor tyrosine kinases: VEGFs, angiopoietins, and ephrins in vascular development. Genes Dev. 13:1055-1066.

6. Jones, N., Iljin, K., Dumont, D.J., and Alitalo, K. 2001. Tie receptors: new modulators of angiogenic and lymphangiogenic responses. Nat. Rev. Mol. Cell Biol. 2:257-267.

7. Lindahl, P., Hellstrom, M., Kalen, M., and Betsholtz, C. 1998. Endothelial-perivascular cell signaling in vascular development: lessons from knockout mice. Curr. Opin. Lipidol. 9:407-411.

8. Betsholtz, C., Karlsson, L., and Lindahl, P. 2001. Developmental roles of platelet-derived growth factors. Bioessays. 23:494-507.

9. Dumont, D.J., Yamaguchi, T.P., Conlon, R.A., Rossant, J., and Breitman, M.L. 1992. tek, a novel tyrosine kinase gene located on mouse chromosome 4 , is expressed in endothelial cells and their presumptive precursors. Oncogene. 7:1471-1480.

10. Dumont, D.J., Gradwohl, G.J., Fong, G.H., Auerbach, R., and Breitman, M.L. 1993. The endothelial-specific receptor tyrosine kinase, tek, is a member of a new subfamily of receptors. Oncogene. 8:1293-1301.

11. Sato, T.N., Qin, Y., Kozak, C.A., and Audus, K.L. 1993. Tie-1 and tie-2 define another class of putative receptor tyrosine kinase genes expressed in early embryonic vascular system. Proc. Natl. Acad. Sci. USA. 90:9355-9358. 
12. Davis, S., et al. 1996. Isolation of angiopoietin-1, a ligand for the TIE2 receptor, by secretion-trap expression cloning. Cell. 87:1161-1169.

13. Holmgren, L., Glaser, A., Pfeifer-Ohlsson, S., and Ohlsson, R. 1991. Angiogenesis during human extraembryonic development involves the spatiotemporal control of PDGF ligand and receptor gene expression. Development. 113:749-754.

14. Lindahl, P., Johansson, B.R., Leveen, P., and Betsholtz, C. 1997. Pericyte loss and microaneurysm formation in PDGF-B-deficient mice. Science. 277:242-245.

15. Hellstrom, M., Kalen, M., Lindahl, P., Abramsson, A., and Betsholtz, C. 1999. Role of PDGF-B and PDGFR-beta in recruitment of vascular smooth muscle cells and pericytes during embryonic blood vessel formation in the mouse. Development. 126:3047-3055.

16. Dumont, D.J., et al. 1994. Dominant-negative and targeted null mutations in the endothelial receptor tyrosine kinase, tek, reveal a critical role in vasculogenesis of the embryo. Genes Dev. 8:1897-1909.

17. Sato, T.N., et al. 1995. Distinct roles of the receptor tyrosine kinases Tie-1 and Tie-2 in blood vessel formation. Nature. 376:70-74.

18. Suri, C., et al. 1996. Requisite role of angiopoietin-1, a ligand for the TIE2 receptor, during embryonic angiogenesis. Cell. 87:1171-1180.

19. Patan, S. 1998. TIE1 and TIE2 receptor tyrosine kinases inversely regulate embryonic angiogenesis by the mechanism of intussusceptive microvascular growth. Microvasc. Res. 56:1-21.

20. Leveen, P., et al. 1994. Mice deficient for PDGF B show renal, cardiovascular, and hematological abnormalities. Genes Dev. 8:1875-1887.

21. Soriano, P. 1994. Abnormal kidney development and hematological disorders in PDGF beta-receptor mutant mice. Genes Dev. 8:1888-1896.

22. Hellstrom, M., et al. 2001. Lack of pericytes leads to endothelial hyperplasia and abnormal vascular morphogenesis. J. Cell Biol. 153:543-554.

23. Folkman, J., and D'Amore, P.A. 1996. Blood vessel formation: what is its molecular basis? Cell. 87:1153-1155.

24. Puri, M.C., Partanen, J., Rossant, J., and Bernstein, A. 1999. Interaction of the TEK and TIE receptor tyrosine kinases during cardiovascular development. Development. 126:4569-4580.

25. Jones, N., et al. 2001. Rescue of the early vascular defects in Tek/Tie2 null mice reveals an essential survival function. EMBO Rep. 2:438-445.

26. Stone, J., and Maslim, J. 1997. Mechanisms of retinal angiogenesis. Progress in retinal and eye research. 16:157-181.

27. Sano, H., et al. 2001. Functional blockade of platelet-derived growth factor receptor-beta but not of receptor-alpha prevents vascular smooth muscle cell accumulation in fibrous cap lesions in apolipoprotein E-deficient mice. Circulation. 103:2955-2960.

28. Yano, M., et al. 1997. Expression and function of murine receptor tyrosine kinases, TIE and TEK, in hematopoietic stem cells. Blood. 89:4317-4326

29. Takakura, N., et al. 1998. Critical role of the TIE2 endothelial cell receptor in the development of definitive hematopoiesis. Immunity. 9:677-686

30. Maisonpierre, P.C., et al. 1997. Angiopoietin-2, a natural antagonist for Tie2 that disrupts in vivo angiogenesis. Science. 277:55-60.

31. Adachi, S., Yoshida, H., Kataoka, H., and Nishikawa, S. 1997. Three distinctive steps in Peyer's patch formation of murine embryo. Int. Immunol. 9:507-514.

32. Fujiwara, T., and Uehara, Y. 1992. The cytoarchitecture of the medial layer in rat thoracic aorta: a scanning electron-microscopic study. Cell Tissue Res. 270:165-172.

33. Gavrieli, Y., Sherman, Y., and Ben-Sasson, S.A. 1992. Identification of programmed cell death in situ via specific labeling of nuclear DNA fragmentation. J. Cell Biol. 119:493-501.

34. Benjamin, L.E., Hemo, I., and Keshet, E. 1998. A plasticity window for blood vessel remodeling is defined by pericyte coverage of the preformed endothelial network and is regulated by PDGF-B and VEGF. Development. 125:1591-1598.

35. Nehls, V., and Drenckhahn, D. 1991. Heterogeneity of microvascular pericytes for smooth muscle type alpha-actin. J. Cell Biol. 113:147-154

36. Fruttiger, M., et al. 1996. PDGF mediates a neuron-astrocyte interaction in the developing retina. Neuron. 17:1117-1131.

37. Orlidge, A., and D'Amore, P.A. 1987. Inhibition of capillary endothelial cell growth by pericytes and smooth muscle cells. J. Cell Biol. 105:1455-1462.

38. Hirschi, K.K., Rohovsky, S.A., Beck, L.H., Smith, S.R., and D'Amore, P.A. 1999. Endothelial cells modulate the proliferation of mural cell precursors via platelet-derived growth factor-BB and heterotypic cell contact. Circ. Res. 84:298-305.

39. Benjamin, L.E., Golijanin, D., Itin, A., Pode, D., and Keshet, E. 1999. Selective ablation of immature blood vessels in established human tumors follows vascular endothelial growth factor withdrawal. J. Clin. Invest. 103:159-165.

40. Kuwabara, T., and Cogan, D.G. 1963. Retinal vascular patterns. VI. Mural cells of the retinal capillaries. Arch. Ophthalmol. 69:492-502.

41. Vracko, R., and Benditt, E.P. 1970. Capillary basal lamina thickening. Its relationship to endothelial cell death and replacement. J. Cell Biol. 47:281-285.

42. Crosby, J.R., Seifert, R.A., Soriano, P., and Bowen-Pope, D.F. 1998. Chimaeric analysis reveals role of Pdgf receptors in all muscle lineages. Nat. Genet. 18:385-388.

43. Sano, H., et al. 2002. Blockade of platelet-derived growth factor receptor- $\beta$ pathway induces apoptosis of vascular endothelial cells and disrupts glomerular capillary formation in neonatal mice. Am. J. Pathol. 161:135-143.

44. Stalmans, I., et al. 2002. Arteriolar and venular patterning in retinas of mice selectively expressing VEGF isoforms. J. Clin. Invest. 109:327-336. doi:10.1172/JCI200214362

45. Fruttiger, M. 2002. Development of the mouse retinal vasculature: angiogenesis versus vasculogenesis. Invest. Ophthalmol. Vis. Sci. 43:522-527.

46. Hirschi, K.K., and D'Amore, P.A. 1996. Pericytes in the microvasculature. Cardiovasc. Res. 32:687-698.

47. Darland, D.C., and D'Amore, P.A. 1999. Blood vessel maturation: vascular development comes of age. J. Clin. Invest. 103:157-158.

48. Hackett, S.F., et al. 2000. Angiopoietin 2 expression in the retina: upregulation during physiologic and pathologic neovascularization. J. Cell. Physiol. 184:275-284.

49. Thurston, G., et al. 1999. Leakage-resistant blood vessels in mice transgenically overexpressing angiopoietin-1. Science. 286:2511-2514.

50. Thurston, G., et al. 2000. Angiopoietin-1 protects the adult vasculature against plasma leakage. Nat. Med. 6:460-463.

51. Hanahan, D. 1997. Signaling vascular morphogenesis and maintenance. Science. 277:48-50.

52. Xu, Y., and Yu, Q. 2001. Angiopoietin-1, unlike angiopoietin-2, is incorporated into the extracellular matrix via its linker peptide region. J. Biol. Chem. 276:34990-34998.

53. Procopio, W.N., Pelavin, P.I., Lee, W.M.F., and Yeilding, N.M. 1999. Angiopoietin-1 and - 2 coiled coil domains mediate distinct homooligomerization patterns, but fibrinogen-like domains mediate ligand activity. J. Biol. Chem. 274:30196-30201.

54. Carlson, T.M., Feng, Y., Maisonpierre, P.C., Mrksich, M., and Morla, A.O. 2001. Direct cell adhesion to the angiopoietins mediated by integrins. J. Biol. Chem. 276:26516-26525.

55. Suri, C., et al. 1998. Increased vascularization in mice overexpressing angiopoietin-1. Science. 282:468-471.

56. Joussen, A.M., et al. 2002. Suppression of diabetic retinopathy with angiopoietin-1. Am. J. Pathol. 160:1683-1693 